\title{
Influence of Thermal Loading on the Dynamic Response of Thin-Walled Structure under Thermo-Acoustic Loading
}

\author{
Yundong Sha ${ }^{1, a}$, Zhijun Gao ${ }^{1, b}$, Fei Xu ${ }^{1}$ and Jiyong $\mathrm{Li}^{1}$ \\ ${ }^{1}$ Shenyang Aerospace University, Liaoning Key Laboratory of Digital Technology Simulation and \\ Test Techniques, Shenyang, Liaoning, 110136, P.R. China \\ aydsha2003@vip.sina.com, ${ }^{b}$ realgaozj@163.com
}

Keyword: Thin-Walled Structure, Dynamic Response, Thermal Buckling, Snap-Through

\begin{abstract}
Future flight vehicle structures will encounter severe loading conditions, a combination of aerodynamic, thermal, acoustic and mechanical loads. Although the analysis methods for responses of structures under acoustic loads have been developed to some extent, but with thermal loads considered, the responses show fundamental differences, which complicate the analysis immensely. It was reported that hypersonic flight may give rise to surface temperature as high as $3000^{\circ} \mathrm{F}$ and intense noise whose overall sound pressure level (OSPL) may reach 180dB. Thin-walled structures subjected to such loadings will exhibit nonlinear responses. Large temperature increments may cause thermal buckling, large thermal deflections and large thermal stresses superimposed on dynamic stresses, coupled with changes in material properties. Both the geometry change by thermal buckling and stiffness change by thermal stress account for the changes of natural frequencies and mode shapes. When the acoustic loading increases to a high enough level, the post-buckled structures will exhibit snap-through motion, a large amplitude nonlinear vibration between different equilibrium positions, which will introduce extra large mean stress. As a result, thermo-acoustic fatigue may be caused, which will reduce the structure's fatigue life dramatically. Therefore it is an urgent need to estimate the influences of thermal loads on the nonlinear response of structures.

A numerical investigation of the influences of thermal loads on the dynamic response of thin-walled structure under thermo-acoustic loadings is implemented. With clamped-clamped thin flat plate selected, the response characteristics related to temperature are investigated by changing thermal loads. The thermal load is considered as constant both on the surface and across the thickness. The acoustic load is simulated using stationary Gaussian white noise. Firstly, a thermal buckling analysis is proceeded to obtain critical buckling temperatures, followed by modal analysis under different thermal loads. The pre-buckled and post-buckled mode frequencies and shapes are obtained. Then three types of snap-though motions are predicted: i) vibration around one post-buckled equilibrium position, ii) intermittent snap-through, and iii) persistent snap-through.

The relations between thermal loads and the occurrence of snap-though is obtained together with results about the statistics characteristic of dynamic response and their relations with thermal loads, which include critical thermal buckling loads, natural frequencies and mode shapes, RMS response and snap-through frequency. Good agreements have been achieved with previous analytical solutions, which demonstrate the effectiveness and reliability of the method employed.
\end{abstract}

\section{Introduction}

The large deflection dynamic response of future flight vehicle structures under thermo-acoustic loadings can lead to significant reduction in fatigue life, which has aroused the interests of many researchers. Several methods have been developed to resolve these problems, including Fokker-Planck-Kolmogorov (FPK) equation, Monte Carlo simulation, Equivalent Linearization (EL) technique, Finite Element method (FEM), etc. The use of Galerkin's approach for solving partial differential governing equation limits the applicability of Monte Carlo simulation only to simple structures [1,2]. Equivalent Linearization method has good accuracy only when the nonlinearity is weak and the response is Gaussian [3]. The drawback of the Finite element method is heavy computational burden. FPK equation yields an exact solution only for single-degree-of-freedom 
(SDOF) system [4,5]. In spite of this, FPK equation provides an easy understanding of thermo-acoustic response and snap-through. In the following sections, the basic characteristics of thermo-acoustic response are analyzes briefly with SDOF equation and potential energy curves, further more, the thermo-acoustic responses of a clamped-clamped rectangular plate under different temperatures and sound pressure levels (SPLs) are simulated by FEM. The numerical results are compared with the theoretical analysis results.

\section{Large Deflection Governing Equation of Plate under Thermo-Acoustic Loadings}

The strain-stress relation with thermal expansion considered takes the forms below:

$$
\varepsilon_{x}=\frac{1}{E}\left(\sigma_{x}-\mu \sigma_{y}\right)+\alpha T \quad \varepsilon_{y}=\frac{1}{E}\left(\sigma_{y}-\mu \sigma_{x}\right)+\alpha T \quad \varepsilon_{x y}=\frac{1+\mu}{E} \sigma_{x y}
$$

where $E$ is Young's modulus, $\mu$ is Poisson ratio, $\alpha$ is coefficient of thermal expansion, $T(x, y, z)$ is the temperature distribution function throughout the plate.

According to thin plate theory, complicated nonlinear temperature variation across the thickness can be ignored. Therefore, assume that the temperature variation across the thickness is linear, which can be written as:

$$
T(x, y, z)=\bar{T}(x, y)+z \theta(x, y)
$$

where $\bar{T}=\frac{1}{h} \int_{-h / 2}^{h / 2} T(z) \mathrm{d} z$ is the temperature averaged across the plate thickness, $h$ is plate thickness, $\theta$ is the thermal gradients across $h$.

With Airy stress function introduced, compatibility equation can be expressed as follows:

$$
\nabla^{4} F+E h \alpha \nabla^{2} \bar{T}=E h\left[\left(\frac{\partial^{2} w}{\partial x \partial y}\right)^{2}-\left(\frac{\partial^{2} w}{\partial x^{2}}\right)\left(\frac{\partial^{2} w}{\partial y^{2}}\right)\right]
$$

where the complete solution of Airy stress function comprises harmonic solution $F_{h}$ and particular solution $F_{p} . w$ is the deflection function of the plate.

The well-known Von Karman large deflection equation with thermal component can be derived:

$$
\rho h \frac{\partial^{2} w}{\partial t^{2}}+\rho h \xi \frac{\partial w}{\partial t}-p(x, y, t)+D \nabla^{4} w+\alpha(1+\mu) D \nabla^{2} \theta=\frac{\partial^{2} w}{\partial x^{2}} \frac{\partial^{2} F}{\partial y^{2}}+\frac{\partial^{2} w}{\partial y^{2}} \frac{\partial^{2} F}{\partial z^{2}}-2 \frac{\partial^{2} w}{\partial x \partial y} \frac{\partial^{2} F}{\partial x \partial y}(4)
$$

where $\rho$ is mass density, $\xi$ is damping coefficient, $p(\mathrm{x}, \mathrm{y}, \mathrm{t})$ is the random pressure used to simulate acoustic loading, $D$ is flexural rigidity, $\nabla^{4}$ is bi-harmonic operator.

Immovable boundary condition Eq. 5 is suitable for both simply supported and clamped rectangular plate, with which $F_{h}$ can be derived.

$$
\begin{cases}\frac{\partial F^{2}}{\partial x \partial y}=0 & \int_{0}^{a} \int_{0}^{b}\left[\frac{1}{E h}\left(\frac{\partial^{2} F}{\partial y^{2}}+\mu \frac{\partial^{2} F}{\partial x^{2}}\right)+\alpha \bar{T}(x, y)-\frac{1}{2}\left(\frac{\partial w}{\partial x}\right)^{2}\right] \mathrm{d} x \mathrm{~d} y=0 \quad x=0, a \\ \frac{\partial F^{2}}{\partial x \partial y}=0 & \int_{0}^{a} \int_{0}^{b}\left[\frac{1}{E h}\left(\frac{\partial^{2} F}{\partial x^{2}}+\mu \frac{\partial^{2} F}{\partial y^{2}}\right)+\alpha \bar{T}(x, y)-\frac{1}{2}\left(\frac{\partial w}{\partial y}\right)^{2}\right] \mathrm{d} x \mathrm{~d} y=0 \quad y=0, b\end{cases}
$$

For plate with uniform temperature distribution, $\bar{T}$ is constant, $\theta=0$, Eq. 3 can be written as:

$D \nabla^{4} w=-\frac{E \alpha h}{1-v} \theta\left(\frac{\partial^{2} w}{\partial x^{2}}+\frac{\partial^{2} w}{\partial y^{2}}\right)$

The deflection function for simply supported plate can be expressed with trigonometric functions:

$$
\mathrm{w}(x, y, t)=\sum_{m=1}^{\infty} \sum_{n=1}^{\infty} A(t) \sin \frac{m \pi x}{a} \sin \frac{n \pi y}{b}
$$


Subscribe Eq. 7 into equation Eq. 6:

$$
D\left[\left(\frac{m \pi}{a}\right)^{2}+\left(\frac{n \pi}{b}\right)^{2}\right]=\frac{E \alpha h \theta}{1-\mu}
$$

The first order critical buckling load for simply-supported plate can be obtained:

$$
T_{s}^{*}=\frac{\pi^{2} h^{2}}{12(1+\mu) \alpha b^{2}}\left(1+\frac{b^{2}}{a^{2}}\right)
$$

The coefficient of thermal buckling $\mathrm{S}$ is introduced to describe the temperature of structures under thermal loading, whose definition is $S=T / T_{c r}$, where $T$ is plate temperature, $T_{c r}$ is critical buckling temperature.

For plate with immovable boundary condition, F can be derived. By employing Galerkin's approach, the partial differential equation Eq. 4 can be reduced to ordinary differential equations in modal co-ordinates.

In order to illustrate and explain the basic characteristic of snap-through, the non-dimensional single-mode plate equation can be derived:

$$
\ddot{q}+\omega_{0} \xi \dot{q}+\omega_{0}^{2}(1-s) q+k q^{3}=f
$$

where $k=3\left[\left(1-\mu^{2}\right)\left(1+b^{4} / a^{4}\right)+2\left(b^{4} / a^{4}+2 \mu b^{2} / a^{2}+1\right)\right], \omega_{0}=\left(a^{2}+b^{2}\right) / a^{2}, s=T / T_{c r}, f=P_{s}$.

The potential energy of the single-mode plate can be written as:

$$
U=\omega_{0}^{2}(1-s) q^{2} / 2+k q^{4} / 4
$$

When $\beta=1, \mu=0.31$, the potential energy curves at $\mathrm{S}=0,1,3,6$ are plotted versus modal displacement in Fig. 1. The snap-through response can be regarded as a result of a qualitative change in the shape of potential energy wells. The potential energy curve of a post-buckled structure consists of two potential wells representing two stable equilibrium positions divided by an unstable position which is the initial state. The snap-through response can be viewed as a reciprocating movement between energy wells, and affected by the depth of the wells and excitation levels. When $S=0$, the potential curve of pre-buckled plate has only one potential well, and the plate tends to oscillate about the lowest point while excited. When $\mathrm{S}=3$, the buckled structure will have two separated potential wells which are shallow and close to each other. If the excitation level is weak, the structure will only vibrate in one of the wells. If excited, the structure may escape from its initial well, and jump into the other. When loaded with high enough random excitation, the structure will exhibit snap-through and keep jumping between two wells. When the temperature goes up to $S=6$ and the excitation level maintained, the depth of the well will increase and the frequency of snap-through will decrease [6,7].

\section{Numerical Results of a Clamped-Clamped Plate}

In this section, a thin-walled flat plate with dimension of $a=0.5 \mathrm{~m}, b=0.05 \mathrm{~m}, \mathrm{~h}=0.002 \mathrm{~m}$ is modeled, and the clamped-clamped boundary condition is applied. The material properties are chosen as follows, the Young's modulus $\mathrm{E}=0.73 \mathrm{GPa}$, the density $\rho=2700 \mathrm{~kg} / \mathrm{m}^{3}$, the coefficient of thermal expansion is $\alpha=2.4 \times 10^{-5} \mathrm{~m} / \mathrm{K}$, the Poisson's ratio is $\mu=0.32$.

The thermal buckling analysis is firstly conducted. The first 10 buckling temperatures are obtained as well as the first 10 modal frequencies under different temperatures, from which the influence of temperature on structural stiffness can be seen, as shown in Fig. 2. An obvious decline of modal frequencies before buckling temperature shows the decrease of stiffness due to compressive thermal stress. After buckling temperature, the post-buckled plate resumes its stiffness due to the change of geometry. It is found that the orders of modes may change, which is called mode shifting, making the thermo-acoustic response more complicated. Based on this, it is believed that the assumptions of linear superposition of linear modes employed in several works may bring about enormous errors at high temperatures $[1,2,6]$. 
This plate is then loaded with thermal load and acoustic load simultaneously. The acoustic load level remains unchanged for different thermal loads. The acoustic load is assumed to be band limited Gaussian white noise. The overall sound pressure level (OSPL) is 160dB, which is demonstrated to be sufficient to induce snap through, and the cut-off frequency is $1500 \mathrm{~Hz}$, which has covered the first 10 modal frequencies, enabling multi-mode participation. Furthermore, the fluctuating sound pressure is assumed to be uniformly distributed on the surface. The thermal load is assumed to be uniformly distributed on the surface and have no gradient across the thickness, ranging from $0^{\circ} \mathrm{C}(S=0)$ to $110^{\circ} \mathrm{C}(S=49.6)$.

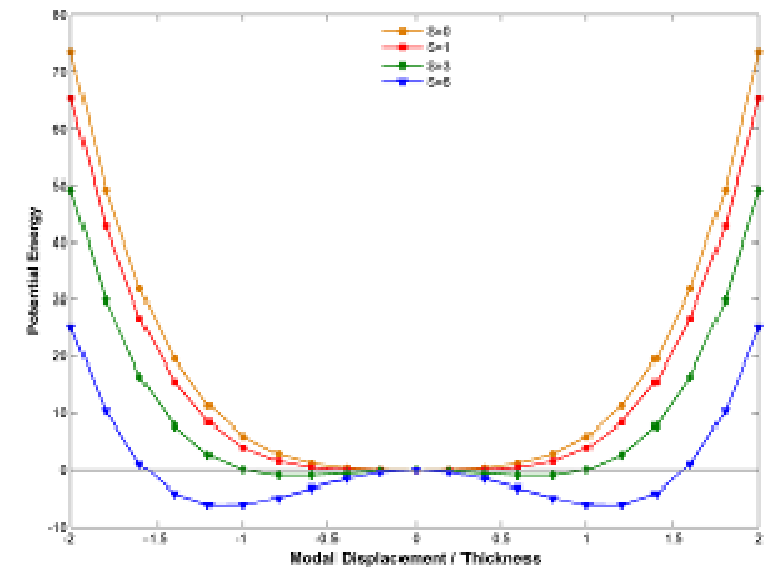

Fig. 1. Potential Energy Curves under Different Temperature

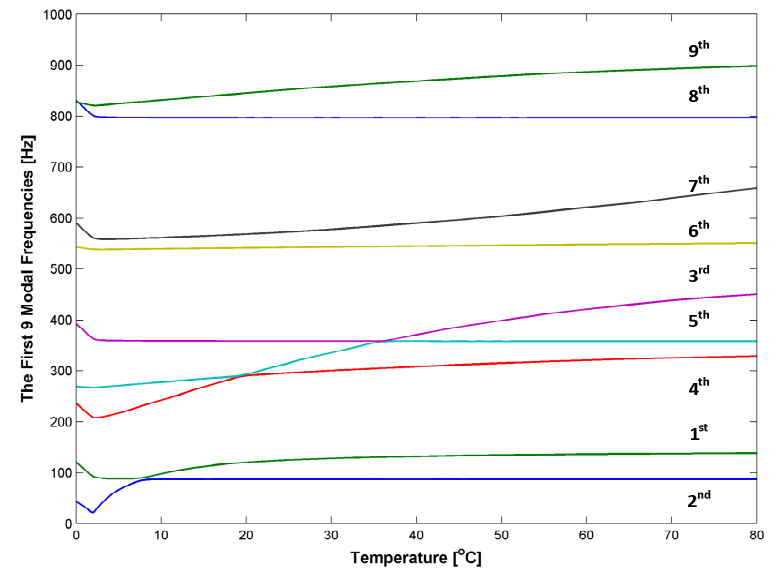

Fig. 2. The First 9 Modal Frequencies as a Function of Temperature

Table 1 The First 10 Critical Temperatures of Clamped-Clamped Plate

\begin{tabular}{c|cccccccccc}
\hline Order & 1 & 2 & 3 & 4 & 5 & 6 & 7 & 8 & 9 & 10 \\
\hline$T_{c r}\left[{ }^{\circ} C\right]$ & 2.2 & 4.6 & 9.0 & 13.7 & 20.7 & 28.1 & 37.9 & 48.2 & 61.1 & 74.7 \\
\hline
\end{tabular}

A series of dynamic responses of the structure under different thermal loads for given acoustic load are obtained. All types of thermo-acoustic response, random vibration, persistent snap-through, intermittent snap-through and small vibration around one post-buckled equilibrium position are observed in sequence as the temperature increases. But there seem no obvious boundaries between these different types of motions. When $S<1$, the pre-buckled structure vibrates around its single equilibrium position, Fig.3. When the temperature increases, $\mathrm{S}>1$, the post-buckled plate becomes curved with two equilibrium positions. If this temperature is still low, the two equilibrium positions are so close that the acoustic excitation needed to snap is low and the snap-through motion under an intense acoustic load is frequent and difficult to identity from normal random vibration. For simplicity, the times of vibrating through 0 point are considered to be times of snap-through motion.

When the temperature increases further, the increase of the static deflection makes the excitation needed to snap goes up. If the plate fails to jump into its opposite potential wells, a horizontal line can then be observed, Fig.4.

When the temperature goes far beyond the critical temperature $S>>1$, the potential well is deep enough and the equilibrium positions are far apart that the snap-through becomes rare, Fig. 5. Finally, the plate can only vibrate around one equilibrium position, Fig. 6.

Although identifying the boundary of snap-through in the time domain is not easy, but from frequency domain it is much easier. It is noted that when snap-through motion occurs, there exists a peak below the fundamental frequency. And as temperature goes up, the peak goes up accordingly and gets close to zero frequency. Because of the capability of real time frequency analysis of present signal processing system, it is a practical method to identify snap-through in frequency domain.

After simple mathematical processing of lateral displacement time history, more interesting details about snap-through motion are revealed. The curves for absolute mean, RMS, variance of midpoint lateral displacement are plotted against temperature, as shown in Fig. 7(a),(b),(c). Although the mean, RMS, and variance go up with temperature generally. But there are several valleys and peaks on the curve. After comparison, it is found that the peaks and valleys are related to critical temperatures. When the temperature gets close to critical temperatures, corresponding modal 
stiffness will decrease, and the participation of corresponding mode will increase. If this mode is symmetric, the midpoint displacement and its mean will increase. If this mode is antisymmetric, the midpoint displacement and its mean will decrease. This explanation can well account for the alternate occurrence of peaks and valleys, but need further experimental validation.

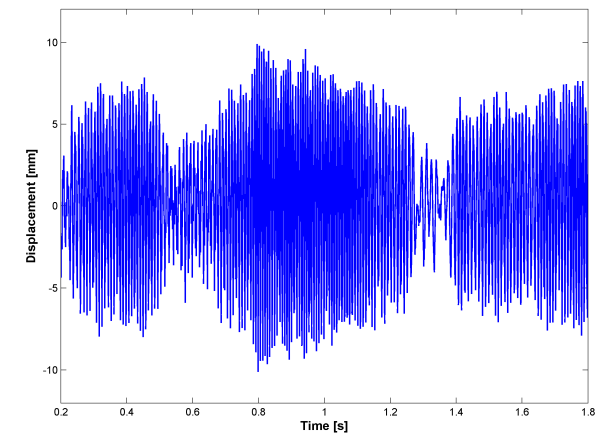

Fig. 3. Random Vibration $\left(\mathrm{T}=0^{\circ} \mathrm{C}, \mathrm{SPL}=160 \mathrm{~dB}\right)$

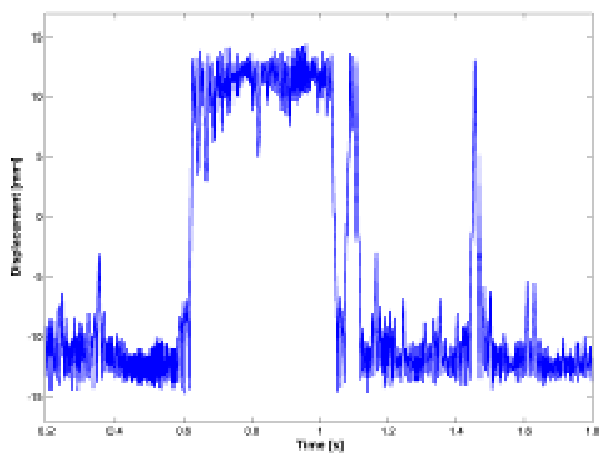

Fig. 5. Intermittent Snap-Through Response $\left(\mathrm{T}=70^{\circ} \mathrm{C}, \mathrm{SPL}=160 \mathrm{~dB}\right)$

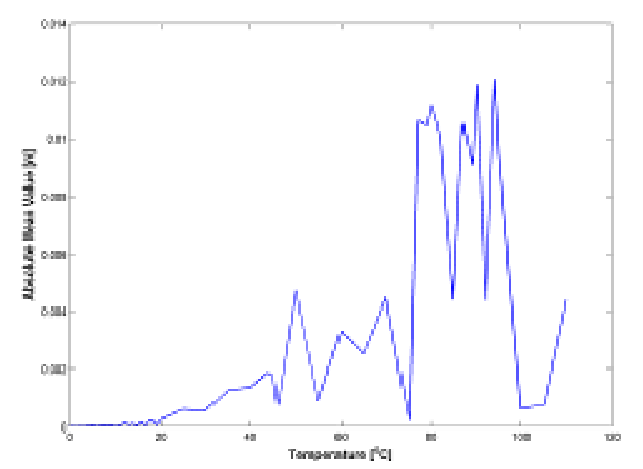

(a) Absolute Mean Value

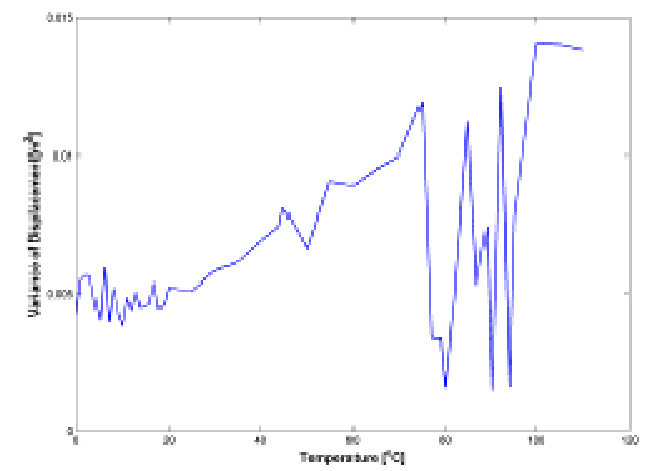

(c) Variance

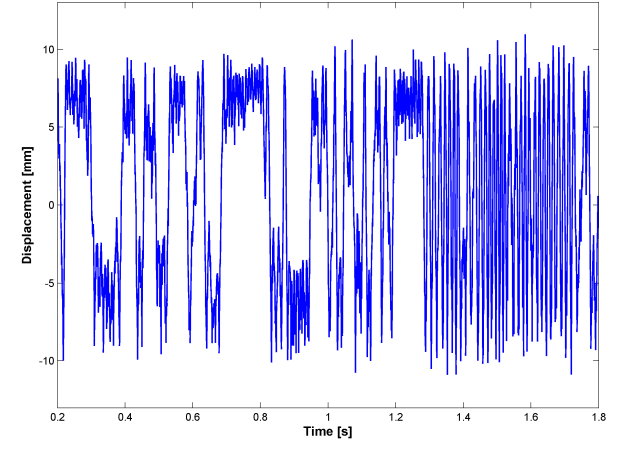

Fig. 4. Persistent Snap-Through Response $\left(\mathrm{T}=30^{\circ} \mathrm{C}, \mathrm{SPL}=160 \mathrm{~dB}\right)$

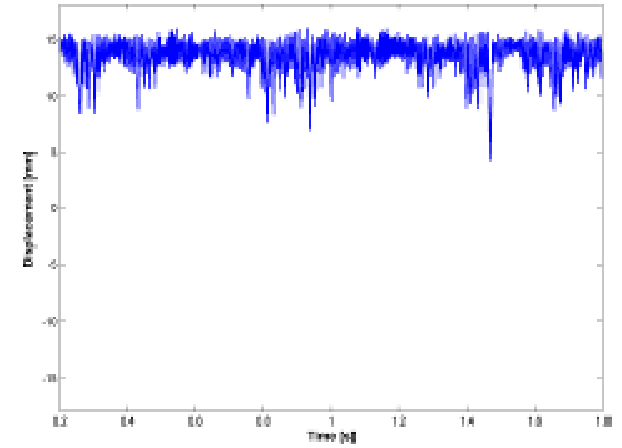

Fig. 6. Vibration around a Post-Buckled Position $\left(\mathrm{T}=90^{\circ} \mathrm{C}, \mathrm{SPL}=160 \mathrm{~dB}\right)$

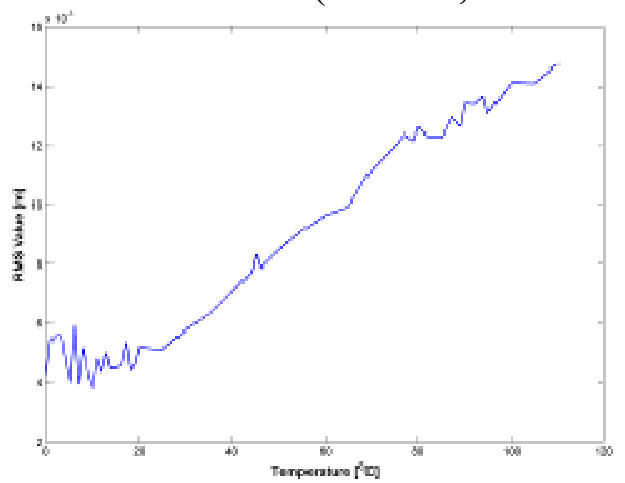

(b) RMS Value

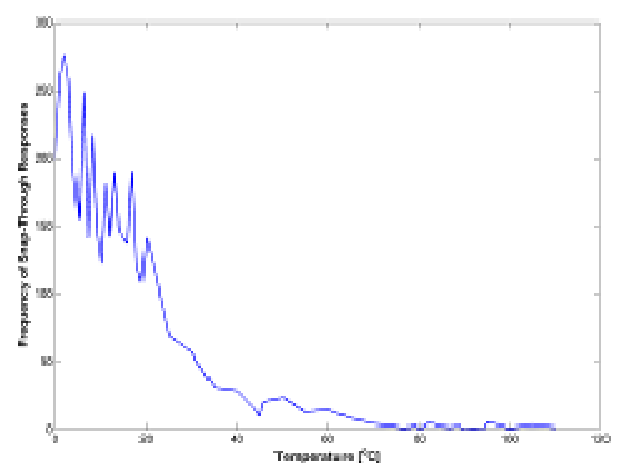

(d) Frequency of Snap-Through

Fig. 7. Midpoint Transverse Displacement as a Function of Temperature (SPL $=160 \mathrm{~dB})$ 


\section{Conclusions}

(1) Thin-walled structures can get buckled at low temperature, no more than several degrees above ambient temperature for some cases. After thermal buckling, post-buckled structures will have multiple equilibrium positions corresponding to multiple potential wells, which make snap-through response possible.

(2) The identification of the boundary of snap-through according to displacement time history is difficult for the two equilibrium positions are close when the temperature has just exceeded the $1 \mathrm{st}$ order critical buckling temperature. It is proposed that the occurrence of snap-through response can be observed by frequency domain analysis, which can be indicated by a peak value near zero frequency.

(3) As the temperature goes up, both the static deflection of post-buckled structure and the depth of potential wells increase. With the acoustic load unchanged, snap-through response becomes less frequent generally. The structure will experience successively persistent snap-through, intermittent snap-through, and vibration around one post-buckled equilibrium position. When thermal load increases, acoustic load must be intensified to maintain the frequency of snap-through response and overcome the increased static deflection.

(4) When the temperature gets close to critical temperatures, the stiffness will be weakened and the participation of corresponding buckling modes will increase. If the corresponding mode shape is symmetric, the midpoint displacement will increase. If this mode shape is antisymmetric, the midpoint displacement will decrease.

(5) When the thermal load is low, thin-walled structures will experience frequent large amplitude stress cycles. When the temperature is high, structures will experience large mean stress cycles. Both of the two cases can affect fatigue life of structures greatly, and need further investigations.

\section{Reference}

[1] Vaicaitis, R., Nonlinear response - A time domain approach C. Aeroacoustics Conference, 10th (1986) 10

[2] Vaicaitis, R., Nonlinear response and sonic fatigue of National Aerospace Space Plane surface panels, J. Journal of Aircraft (1994) 10-18

[3] Rizzi, S.A. and Muravyov, A.A., Comparison of nonlinear random response using equivalent linearization and numerical simulation, C. Structural Dynamics: Recent Advances, Proceedings of the 7th International Conference (2000) 833-846

[4] Lee, J., Displacement and strain histograms of thermally buckled composite plates in random vibration, C. AIAA/ASME/ASCE/AHS/ASC Structures, Structural Dynamics, and Materials Conference and Exhibit, 37 th (1996) 259-266

[5] Wang, R., Yasuda, K., et al., A generalized analysis technique of the stationary FPK equation in nonlinear systems under Gaussian white noise excitations, J. International Journal of Engineering Science (2000) 1315-1330

[6] Murphy, K.D., Virgin, L.N., et al., Characterizing the dynamic response of a thermally loaded, acoustically excited plate, J. Journal of Sound and Vibration (1996) 635-658

[7] Thompson, J.M.T., Chaotic phenomena triggering the escape from a potential well, J. Proceedings of the Royal Society of London. A. Mathematical and Physical Sciences (1989) 195 